INRA Prod. Anim., 2000, 13 (4), 223-231
J. THIMONIER ${ }^{1}, Y_{\text {. COGNIE }}^{2}$,

N. LASSOUED ${ }^{3}$, G. KHALDI $^{4}$

1 ENSA.M-INRA, UMR Elevage des Rumi nants en Régi ons chaudes, 2 Place Viala, 34060 Montpelli er Cedex 01 2 INRA, UMR Physi ologi e de la Reproduction et des Comportements, 37380 Nouzilly

3 I NRAT, Laboratoi re des Recherches ovi nes et caprines, Rue Hédi Karray, 2049 Ariana, Tunisie

4 Institut Nati onal Agronomi que de Tuni si e, 43 Avenue Charles Ni colle, 1082 Tunis-Mahrajene, Tuni si e

courriel : thi moni e@ensam.inra.fr

\section{L'effet mâle chez les ovins : une technique actuelle de maîtrise de la reproduction}

Les périodes de repos sexuel limitent l'efficacité de la reproduction chez les ovins. Différentes techniques faisant appel à des traitements hormonaux, associés ou non à des traitements lumineux, peuvent être utilisées pour contrôler le moment de la reproduction dans un troupeau. Une méthode alternative, sans utilisation d'hormones, peut également être appliquée avec succès, dans certaines conditions.

La reproduction des brebis est saisonnée ; les oestrus ou chaleurs apparaissent normalement en fin d'été et en automne. Cette période (saison sexuelle) peut être plus ou moins étendue selon les races. Elle est suivie, depuis la fin de l'hiver jusqu'au début de l'été, par une période de repos sexuel appelée anoes-

\section{Résumé}

L'introduction des béliers dans un groupe de brebis en anoestrus anovulatoire induit dans les $\mathbf{2}$ à $\mathbf{4}$ jours une ovulation silencieuse, c'est-à-dire non associée à un comportement de chaleur, suivie soit par un cycle ovulatoire de durée «normale» (voisine de 17 jours) puis d'une nouvelle ovulation associée à un comportement de chaleur, soit par un cycle ovulatoire de durée courte mais constante ( 6 jours) suivi d'une nouvelle ovulation silencieuse puis, après un cycle ovulatoire de durée normale, par une nouvelle ovulation associée à un comportement d'oestrus.

Il en résulte que les premières chaleurs apparaissent très tardivement chez les femelles dont l'activité sexuelle est induite par l'introduction des béliers, ce qui se traduit par deux pics d'activité sexuelle situés environ 18 à 20 jours et 24 à 26 jours après l'introduction des béliers. Les brebis non gravides reviennent normalement en oestrus sauf si l'anoestrus est intense au moment attendu de ces retours (plus de un mois après induction de la première ovulation). L'article présente les connaissances acquises ces dernières années sur les mécanismes physiologiques impliqués dans l'induction de l'ovulation par l'effet bélier et donne quelques règles d'utilisation de cette méthode dans les élevages, tout en précisant ses limites. Les deux points essentiels pour utiliser efficacement cette technique sont d'une part, la connaissance des aptitudes de reproduction (saisonnement) de la race dans son milieu et, d'autre part, la possibilité de séparer les mâles des femelles pendant une durée minimale de un mois préalablement à leur mise en contact physique. trus saisonnier. La durée et l'intensité (certains disent profondeur) de cet anoestrus varient d'une race à l'autre. Alors que dans certaines races, toutes les brebis sont en arrêt simultané de reproduction, chez plusieurs autres dont celles du pourtour méditerranéen, il n'en est pas de même (Thimonier et Mauléon 1969) : pendant I'anoestrus, quelques brebis présentent des chaleurs. D'autres femelles, en anoestrus, ont malgré tout des ovulations dites silencieuses (non associées à un oestrus) qu'il est possible de déceler par observation endoscopique des ovaires ou analyse des niveaux de progestérone périphérique (Thimonier 2000). Cette activité ovulatoire non apparente n'est pas rare en avril-mai chez certaines races.

Ce saisonnement de l'activité sexuelle des femelles, plus ou moins marqué suivant les races, n'est pas leur privilège. Chez les mâles, le poids testiculaire, qui reflète l'activité spermatogénétique, est faible pendant la période qui correspond à celle de moindre activité des femelles et élevé pendant celle correspondant à la saison sexuelle. La sécrétion de testostérone subit également des variations saisonnières importantes qui ont des conséquences sur le comportement sexuel, qui est plus faible au printemps qu'à l'automne (Rouger 1974, Ortavant et al 1988). 
Néanmoins, pendant l'anoestrus, des techniques particulières de conduite du troupeau sont susceptibles de provoquer l'ovulation et l'apparition des chaleurs. Elles ont été présentées dans plusieurs articles de cette revue (Cognié 1988, Chemineau et al 1996 et 1999). La plupart de ces techniques font appel à des traitements hormonaux ou à l'association de traitements photopériodiques et hormonaux. A I'heure actuelle, des methodes alternatives sans utilisation d'hormones sont souhaitées pour mieux répondre aux attentes des consommateurs. L'effet mâle, déjà décrit chez les caprins (Chemineau 1989) et les porcins (Prunier 1989) pour induire une activité de reproduction chez les femelles en anoestrus, est aussi une méthode employée depuis très longtemps chez les ovins puisque la première référence connue date de 1813 (Girard 1813). II est probable qu'une recherche plus approfondie dans des textes plus anciens ferait émerger de l'oubli des références originales. Bien ultérieurement, l'étude de l'effet mâle chez les ovins de la race Mérinos d'Arles, race pour laquelle la lutte de printemps est une règle quasi-générale, a été faite par Prud'hon et collaborateurs (Prud'hon et al 1966 et 1968 Prud'hon et Denoy 1969). Ces auteurs montrent que l'apparition des oestrus au cours de la lutte de printemps n'est pas uniforme, contrairement à ce qui est observé lors d'une lutte d'automne. Ils proposent l'utilisation de béliers vasectomisés pendant les premiers jours de la lutte de printemps avant l'introduction des mâles reproducteurs, pour obtenir un meilleur groupement des oestrus et une fertilité élevée chez les brebis adultes (voisine de $90 \%$ ), plus variable chez les antenaises.

A la fin des années 1970, de nouvelles recherches se sont développées. La spécificité dans les réponses à l'effet mâle est si caractéristique qu'il apparaît opportun de faire le point des connaissances sur l'effet mâle chez les ovins et sur les possibilités de son utilisation dans les élevages.

\section{1 / Principe de l'effet mâle}

Lorsque, après une séparation d'une durée au moins égale à un mois, des béliers sont introduits dans un troupeau de brebis en inactivité ovulatoire, une grande partie des femelles ovulent dans les 2 à 4 jours qui suivent (figure 1). Ce premier moment d'ovulation est silencieux. II peut être suivi directement, environ 17 jours plus tard (la durée d'un cycle

Figure 1. Représentation schématique de la réponse à l'effet mâle chez la brebis.

La partie à gauche de l'ordonnée représente le pourcentage de femelles ovulant spontanément. C'est un indicateur de l'intensité de l'anoestrus. Ce pourcentage est compris entre 0 (anoestrus anovulatoire) et environ 5,9\% (toutes les femelles ayant une activité ovulatoire spontanée : cas de la saison sexuelle). La partie à droite de l'ordonnée ne concerne que les femelles anovulatoires avant l'introduction des mâles. Le pourcentage de ces femelles ovulant dans les 2 à 4 jours après introduction des béliers est fonction de l'intensité de l'anoestrus. Il en est de même du pourcentage de femelles ayant répondu à l'effet mâle qui ont un premier cycle ovulatoire de courte durée. Ainsi, plus l'intensité de l'anoestrus est forte (c'est-àdire plus le pourcentage de femelles ovulant spontanément avant l'introduction des béliers est faible), moins la réponse à l'effet mâle sera forte et plus la proportion de femelles répondant à l'effet mâle ayant un cycle ovulatoire de courte durée sera élevée. Pour les races très saisonnées, la réponse à l'effet mâle peut être nulle à certaines périodes de l'anoestrus.

L'ovulation induite par effet mâle est une ovulation silencieuse. Il en est de même de l'ovulation après un cycle ovulatoire de courte durée. Les premiers oestrus chez les femelles précédemment anovulatoires n'apparaissent donc qu'au-delà du $15^{\text {ème }}$ jour après l'introduction des béliers dans le troupeau.

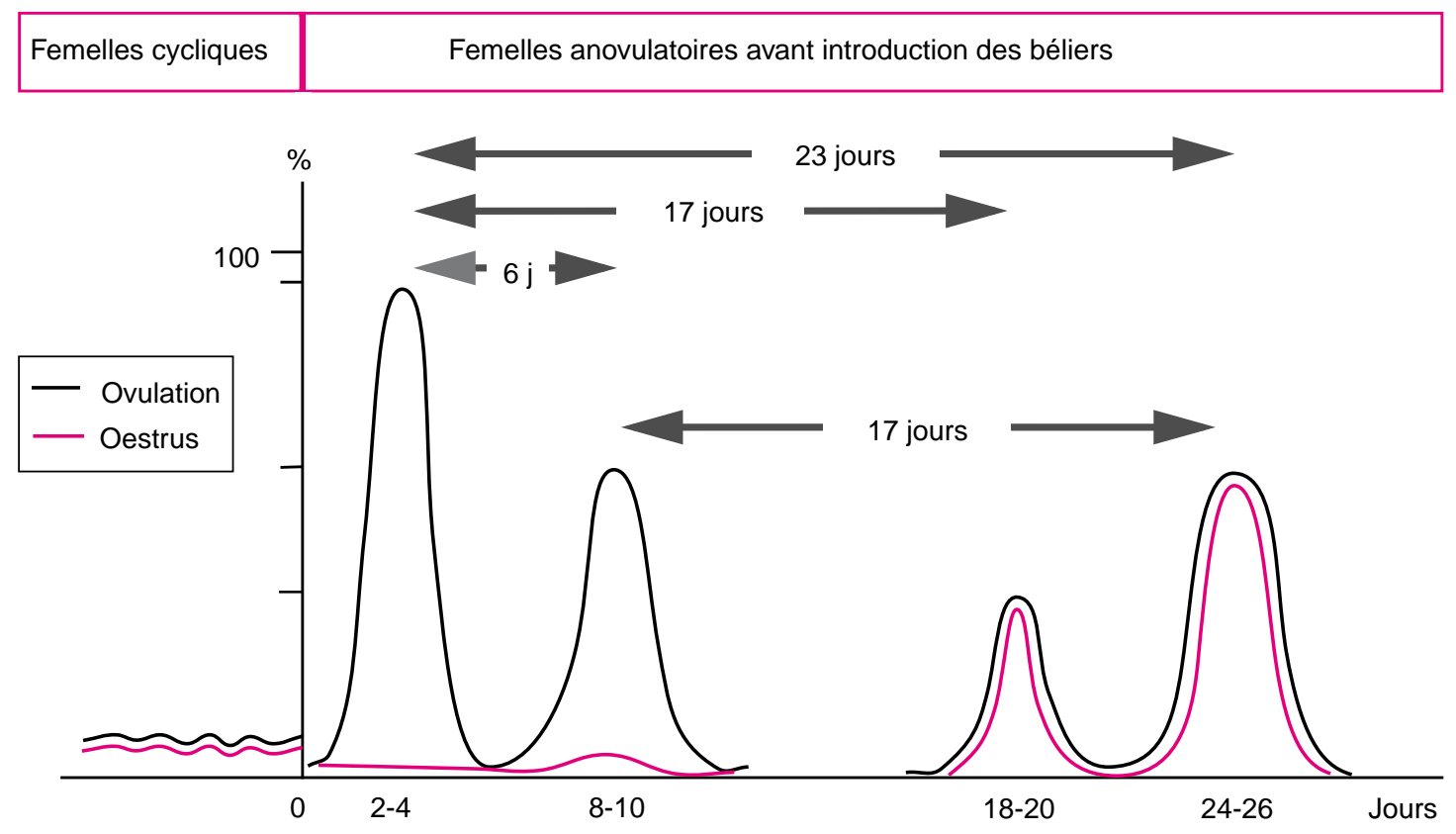

INRA Producti ons Ani males, octobre 2000 
normal chez la brebis), d'un second moment d'ovulation généralement associé à un comportement de chaleur. Cependant, dans certains cas dont la fréquence est variable, ce premier moment d'ovulation est suivi d'un cycle ovulatoire de durée courte mais relativement constante (environ 6 jours) puis d'un nouveau moment d'ovulation généralement silencieux également. Ce n'est qu'après un deuxième cycle ovulatoire de durée normale qu'apparaissent alors oestrus et ovulation.

Ainsi, dans un troupeau de femelles en anoestrus dans lequel l'effet mâle est pratiqué avec succès, il existe deux pics d'apparition des chaleurs, respectivement $18-20$ jours et $24-26$ jours après introduction des béliers.

En fait, compte tenu de la variabilité de la durée des cycles sexuels (15 à 19 jours), la plupart des brebis seront saillies au cours de la seconde quinzaine de lutte avec une bonne synchronisation.

La proportion des brebis répondant à l'effet mâle et le pourcentage d'entre elles ayant un cycle ovulatoire de courte durée (donc deux moments d'ovulation silencieux) sont fonction de l'intensité de l'anoestrus. Si le repos sexuel, ou anoestrus, est intense (ou profond), peu de brebis ovulent en réponse à l'introduction des béliers dans le troupeau et la plupart de celles qui ovulent ont deux moments d'ovulation silencieux successifs (à un intervalle de 6 jours) avant l'ovulation associée à une chaleur. Au contraire, en cas d'anoestrus moins marqué (des femelles sont donc déjà spontanément ovulatoires), la proportion de brebis ovulant en réponse à l'introduction des béliers sera élevée et les cycles ovulatoires de courte durée seront peu nombreux.

Des retours en chaleur chez les femelles non gravides sont généralement observés sauf si l'intensité de l'anoestrus au moment de ces éventuels retours en chaleur est forte.

\section{1 / Intensité de l'anoestrus et réponse à l'effet mâle}

L'intensité de l'anoestrus est donc un paramètre important permettant de prévoir la réponse à l'effet mâle. Expérimentalement, il est possible d'apprécier l'intensité de l'anoestrus par deux approches différentes

- analyse de la fréquence des décharges pulsatiles de LH (hormone lutéinisante) par mesure des niveaux plasmatiques de LH dans des prélèvements sanguins effectués toutes les 10 ou 15 minutes pendant une période d'une durée au moins égale à 6 heures. Plus la fréquence est élevée, plus l'intensité de l'anoestrus est faible (Poindron et al 1980 ; figure 2) ;

- connaissance du pourcentage de femelles ayant des ovulations spontanées avant l'introduction des mâles. Deux méthodes sont utilisables : analyse des niveaux de progestérone plasmatique périphérique dans des prélèvements sanguins effectués à un intervalle compris entre 8 et 10 jours (Thimonier
Figure 2. Fréquence des décharges pulsatiles de LH chez des brebis anovulatoires avant et après mise en contact avec un bélier (d'après Poindron et al 1980).

Les prélèvements sont effectués toutes les 20 minutes pendant 6 heures ( $A$ et $B$ ) avant et après $(C)$ l'introduction du bélier puis jusqu'à la décharge préovulatoire de $L H(D)$. La fréquence des décharges pulsatiles de LH est faible pendant l'anoestrus. Elle est cependant plus élevée chez les brebis Préalpes que chez les brebis lle de France, ce qui reflète la différence d'intensité de l'anoestrus entre les deux races (Thimonier et Mauléon 1969). La mise en contact avec un mâle augmente immédiatement la fréquence des décharges pulsatiles de $\mathrm{LH}$ et conduit à la décharge préovulatoire si le contact physique est maintenu.
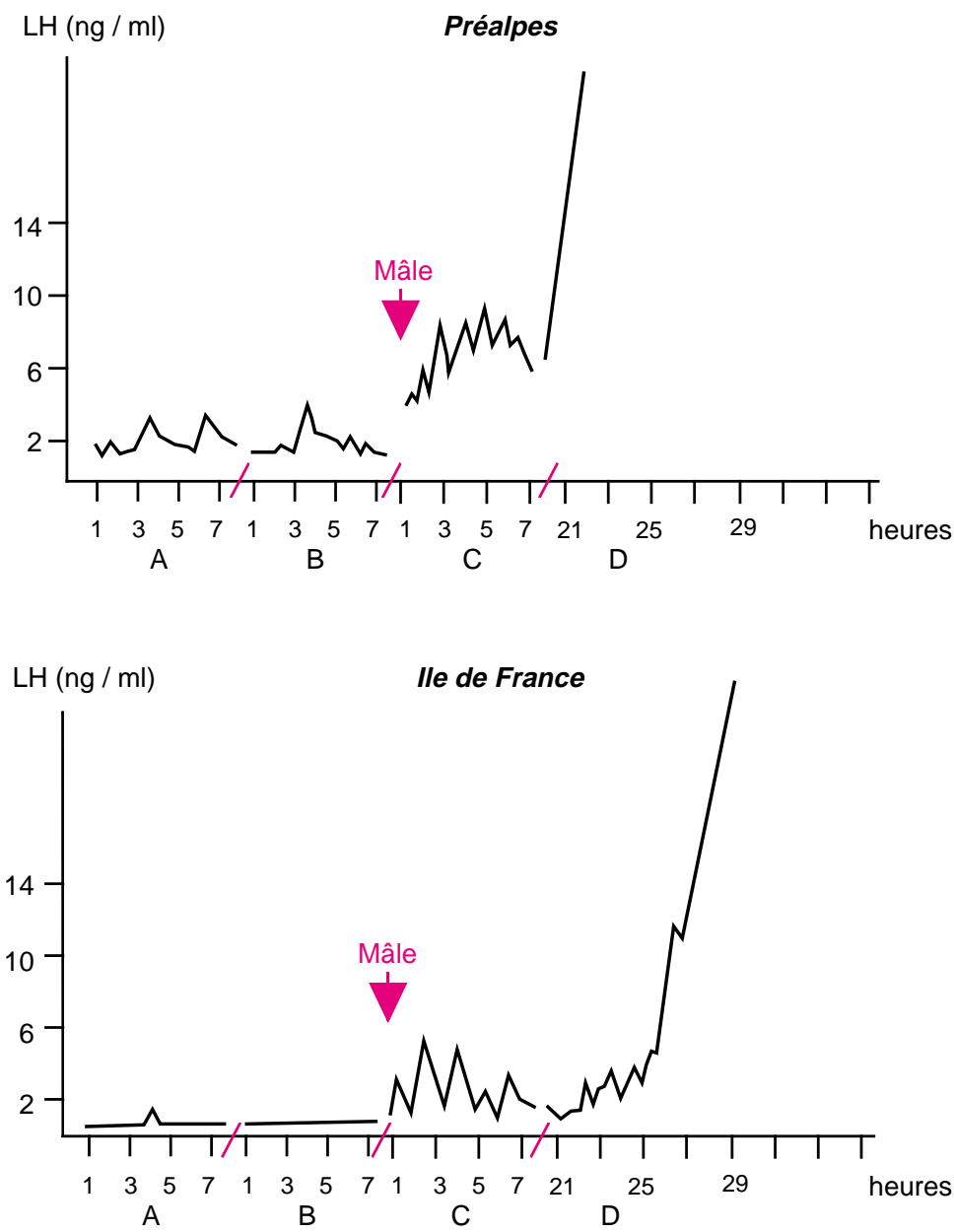

2000) ou observation directe des corps jaunes par endoscopie (Thimonier et Mauléon 1969). Plus la proportion de femelles ayant une activité ovulatoire spontanée est plus élevée, moins l'anoestrus est intense (Signoret 1990).

Ces approches ont été effectuées sur différentes races à différentes périodes et dans différentes conditions d'élevage. Elles sont difficiles à mettre en œuvre par un éleveur. II a ainsi été montré que l'intensité de l'anoestrus varie en fonction de la race mais également avec le moment de la saison d'anoestrus, le niveau nutritionnel, l'état physiologique et l'âge des femelles.

Trois exemples sont donnés pour lesquels les critères retenus sont la proportion de femelles ayant une activité ovulatoire avant l'introduction des mâles, la proportion de 
femelles anovulatoires qui ovulent après introduction des mâles dans le troupeau et la proportion de ces femelles ayant un premier cycle ovulatoire de courte durée. Ainsi, les agnelles et les antenaises répondent moins bien à l'effet mâle que les brebis adultes (tableau 1). Il en est de même des femelles sous-alimentées (tableau 2). Les brebis allaitantes ont un anoestrus dont l'intensité diminue au cours de la pério de post-partum et, par conséquent, la réponse à l'effet mâle s'améliore au fur et à mesure que la date de la mise bas s'éloigne (tableau 3). D'ailleurs, pour la race Barbarine, différentes relations ont été établies par Khaldi et Lassoued (1991) :

- le pourcentage $Y$ de femelles anovulatoires au printemps (fin avril-début mai), qui reflète l'intensité de l'anoestrus, est négativement corrélé à leur poids vif $X$ au moment du tarissement au début du mois de mars :
$Y=-2,57 X+178,5(r=-0,58 ; P<0,05)$

- le pourcentage $Y$ de femelles dont le premier cycle ovulatoire induit par effet mâle est de courte durée (environ 6 jours) est aussi corrélé négativement avec le poids vif $X$ des femelles lors du tarissement:

$Y=-2,48 X+157,6(r=-0,71 ; P<0,01)$

- enfin, le pourcentage $Y$ de femelles dont le premier cycle ovulatoire induit par effet mâle est de courte durée (environ 6 jours) est corrélé positivement au pourcentage $X$ de femelles anovulatoires avant l'introduction des béliers :

$Y=0,39 X+26,9(r=0,49 ; P<0,05)$

A notre connaissance, de telles relations n'ont pas été établies pour d'autres races.

Ainsi peuvent s'expliquer les variations d'une race à une autre, d'une année à l'autre des résultats d'une lutte pratiquée après induction de l'activité sexuelle par effet mâle.

Tableau 1. Réponse, en fonction de l'âge, à l'effet mâle chez les femelles de race Barbarine à queue grasse, fin avril-début mai (G. Khaldi, non publié).

\begin{tabular}{|l|c|c|}
\hline Groupe & Brebis & Antenaises \\
\hline Nombre de femelles & 160 & 40 \\
\% femelles ovulatoires avant introduction des mâles & $50,6 \mathrm{a}$ & $22,5 \mathrm{~b}$ \\
Nombre de femelles non ovulatoires avant introduction des mâles & 79 & 31 \\
\%femelles non ovulatoires ovulant après introduction des mâles & $97,5^{\mathrm{a}}$ & $74,2 \mathrm{~b}$ \\
\%de ces femelles ayant un cycle ovulatoire de courte durée & 23,4 & 34,8 \\
\hline
\end{tabular}

Pour une même ligne, les valeurs affectées de lettres différentes diffèrent significativement $(P<0,05)$.

Tableau 2. Niveaux alimentaires et réponse à l'effet mâle (début mai) chez les brebis de race Barbarine à queue grasse en Tunisie (G. Khaldi, non publié).

$B B=$ maintien d'un poids vif constant faible (environ $39 \pm 2,6 \mathrm{~kg}$ ) tout au long de la période expérimentale (- 9 semaines avant l'introduction des mâles à + 5 semaines après) ; $H H=$ maintien d'un poids vif constant élevé (environ $52,5 \pm 3,5 \mathrm{~kg}$ ) tout au long de la période expérimentale ; $M M=$ maintien d'un poids vif constant moyen (environ $45,8 \pm 1,6 \mathrm{~kg}$ ) tout au long de la période expérimentale. Les femelles $\mathrm{HB}, \mathrm{BH}$ et MM ont le même poids vif moyen (voisin de $45 \mathrm{~kg}$ ) au moment de l'introduction des mâles dans le troupeau.

\begin{tabular}{|l|c|c|c|c|c|}
\hline Groupe & $\mathrm{BB}$ & $\mathrm{BH}$ & $\mathrm{HB}$ & $\mathrm{HH}$ & $\mathrm{MM}$ \\
\hline Nombre de femelles & 25 & 24 & 25 & 24 & 24 \\
\% femelles ovulatoires avant introduction des mâles & $8,0^{\mathrm{a}}$ & $4,2^{\mathrm{a}}$ & $48,0^{\mathrm{b}}$ & $33,3^{\mathrm{b}}$ & $12,5^{\mathrm{ab}}$ \\
Nombre de femelles non ovulatoires avant introduction des mâles & 23 & 23 & 13 & 16 & 21 \\
\% femelles non ovulatoires ovulant après introduction des mâles & $65,2^{\mathrm{a}}$ & $91,3^{\mathrm{b}}$ & $76,9^{\mathrm{ab}}$ & $87,5^{\mathrm{ab}}$ & $90,3^{\mathrm{b}}$ \\
\%de ces femelles ayant un cycle ovulatoire de courte durée & $53,3^{\mathrm{a}}$ & $76,2^{\mathrm{a}}$ & $20,0^{\mathrm{b}}$ & $21,4^{\mathrm{b}}$ & $31,6^{\mathrm{b}}$ \\
\hline
\end{tabular}

Pour une même ligne, les valeurs affectées de lettres identiques ne diffèrent pas significativement à $P<0,05$.

Tableau 3. Réponse à l'effet mâle en fonction de l'intervalle mise bas-introduction des béliers dans le troupeau chez des brebis de race Barbarine à queue grasse ayant mis bas en octobre (G. Khaldi, non publié).

\begin{tabular}{|l|c|c|c|}
\hline Intervalle mise bas - effet mâle & 15 jours & 25 jours & 35 jours \\
\hline Nombre de femelles & 20 & 20 & 20 \\
\%femelles ovulatoires avant introduction des mâles & $0 \mathrm{a}$ & $40 \mathrm{~b}$ & $40 \mathrm{~b}$ \\
Nombre de femelles non ovulatoires avant introduction des mâles & 20 & 12 & 12 \\
\%femelles non ovulatoires ovulant après introduction des mâles & $70,0 \mathrm{a}$ & $91,7 \mathrm{ab}$ & $100,0 \mathrm{~b}$ \\
\%de ces femelles ayant un cycle ovulatoire de courte durée & $71,4 \mathrm{a}$ & $27,3 \mathrm{~b}$ & $25,0^{\mathrm{b}}$ \\
\hline
\end{tabular}

Pour une même ligne, les valeurs affectées de lettres identiques ne diffèrent pas significativement à $P<0,05$. 
L'importance des différents facteurs est connue intuitivement par les éleveurs et les techniciens qui, en fonction de différents paramètres (état corporel, moment de l'année, ... ), apprécient indirectement l'intensité de l'anoestrus.

\section{2 / Mécanismes impliqués dans la réponse à l'effet mâle}

L'introduction des mâles dans le troupeau de femelles anovulatoires est suivie immédiatement par une augmentation de la fréquence des décharges pulsatiles de $\mathrm{LH}$, ce qui conduit, si les mâles sont maintenus dans le troupeau, à une décharge préovulatoire de LH (Poindron et al 1980). C'est un phénomène identique à celui qui est observé chez les caprins (Chemineau 1989). Dans la pratique, les mâles doivent être présents dans le troupeau, en permanence, au moins pendant les 15 premiers jours (Signoret 1990).

Tous les sens de la femelle sont impliqués dans la réponse à l'effet mâle (odorat, vue, ouie, toucher). La réponse ovulatoire maximale est toujours obtenue lorsqu'il y a contact physique entre mâle(s) et femelles (Pearce et Oldham 1988). Cependant I'odorat est aussi très important. Les béliers émettent des phéromones, dont la nature est partiellement connue (Signoret 1990), pas forcément perceptibles par les humains, qui entraînent la pulsatilité de LH et donc la réponse ovulatoire des brebis. Ces phéromones sont sous la dépendance des stéroïdes sexuels : Ies mâles castrés n'induisent pas l'ovulation chez les femelles anovulatoires. En revanche, des mâles ou des femelles castrés recevant un traitement «stéroïdes» sont capables d'induire la réponse ovulatoire. La laine et les sécrétions des glandes sébacées semblent véhiculer le message phéromonal (Knight et Lynch 1980) : I'odeur de la laine, mais pas celle de l'urine, permet d'induire une augmentation des décharges pulsatiles de LH et l'ovulation chez des brebis anovulatoires (Signoret 1990). Des brebis anosmiques (dont l'odorat a été supprimé) répondent à l'effet mâle en présence de mâles entiers, mais ne répondent pas à la seule inhalation des odeurs de la laine. L'extrait de poils de boucs est aussi efficace pour induire la réponse de brebis normales. La réciproque n'est pas aussi vraie : des extraits de laine de béliers n'induisent pas l'ovulation chez la chèvre. II semble bien qu'un «cocktail» de composés soit impliqué, aussi bien chez les ovins (Signoret et al 1997) que chez les caprins (Walkden-Brown et al 1999).

Le fait que les phéromones soient sous influence des sécrétions stéroïdiennes pourrait rendre compte des différences raciales qui ont été notées dans l'aptitude des mâles à induire l'ovulation (Tervit et al 1977, Knight et al 1980, Signoret 1990) ou de l'importance du nombre de mâles nécessaires pour un effet mâle efficace. Les caractéristiques comportementales (libido) des béliers influent sur leur aptitude à stimuler les brebis (Signoret 1990). Chez les caprins, en période de faible activité sexuelle pour les mâles et d'anoestrus chez les femelles, un traitement photopériodique (lumière + mélatonine) uniquement des mâles permet de stimuler leur comportement sexuel et d'accroître considérablement la réponse des femelles à l'effet mâle (Delgadillo ê al 2000). Une réponse identique devrait être obtenue chez les ovins, le même traitement photopériodique induisant une forte croissance testiculaire chez les béliers (Chemineau et al 1992). La réponse à l'effet mâle ne dépend donc pas uniquement des femelles et de l'intensité de leur anoestrus mais aussi et surtout de l'activité sexuelle des mâles.

L'existence de cycles ovariens de très courte durée (environ 6 jours) a été mise en évidence il y a une vingtaine d'années seulement (Oldham et Lindsay 1980) grâce à l'utilisation de l'endoscopie. Au cours de différentes études, il a été montré que l'ablation de l'utérus (hystérectomie) supprime ces cycles courts (Chemineau et al 1993, Lassoued et al 1997). In vi tro, les cellules lutéales de corps jaunes induits par effet mâle produisent moins de progestérone que des cellules lutéales résultant d'une induction par un traitement progestagène. D'ailleurs, les grandes cellules lutéales sont en moindre proportion dans le premier type de corps jaunes que dans le deuxième (Chemineau et al 1993). La cautérisation des follicules deux jours après I'ovulation supprime également les cycles courts. Inversement, chez des femelles ayant subi la cautérisation des follicules, une administration d'oestradiol réinduit l'apparition de cycles ovulatoires de courte durée chez la totalité des femelles. Une sécrétion précoce de prostaglandine $F 2 \alpha$ sous influence oestrogénique, serait responsable de ces cycles ovulatoires de courte durée. L'admi-nistration in utero ou par voie générale d'inhibiteurs de la synthèse de prostaglandine $\mathrm{F} 2 \alpha$ augmente la durée de ces cycles courts (Lassoued đal 1997).

De même, ces cycles courts peuvent être supprimés par une injection de progestérone ou d'un progestagène dans les deux jours qui précèdent l'introduction des mâles (Lindsay et al 1982). Cette suppression pourrait résulter d'une double action de la progestérone: au niveau utérin (Southee et al 1988) et au niveau du ou des follicule(s) oestrogénique(s) (Scaramuzzi et Downing 1999). L'ovulation induite est cependant retardée d'environ 24 heures lorsque cette injection est faite lors de l'introduction des béliers (Cognié 1988, Lassoued et al 1995).

II existe toutefois une différence fondamentale entre une injection ponctuelle de progestérone et un traitement progestagène d'une durée supérieure à 6 jours. Ce dernier permet l'obtention d'oestrus dès la première ovulation induite alors qu'une seule injection de progestérone est insuffisante pour obtenir un tel résultat (Lindsay et al 1982). II est connu (Robinson 1959) que l'imprégnation préalable du système nerveux central par la progestérone 
(ou un progestagène) est nécessaire pour l'obtention d'un comportement d'oestrus lors de l'ovulation chez les ovins. C'est pour cette raison que la première ovulation à la puberté, lors de la reprise de l'activité sexuelle saisonnière ou après la mise bas est pratiquement toujours silencieuse chez les ovins au contraire de ce qui peut être observé pour les caprins, les bovins, les porcins, ...

\section{2 / Utilisation en élevage}

\section{1 / Intérêt}

L'effet mâle est un moyen économique et efficace pour:

- déclencher l'activité sexuelle des brebis en période d'anoestrus, en particulier au printemps, ce qui permet une production d'agneaux à contre-saison avec l'intérêt économique qui s'y attache ;

- synchroniser les accouplements, et, à un moindre degré, les mise bas, ce qui facilite la surveillance des agnelages et la constitution de lots homogènes d'engraissement ;

- améliorer la fertilité du troupeau lorsque la période de lutte de printemps est de courte durée (cas des troupeaux effectuant une transhumance : Mérinos d'Arles, par exemple). Dans ce cas, I'utilisation de béliers vasectomisés ou munis de tabliers, quinze jours avant la mise en place des béliers de service, avance d'autant l'apparition des chaleurs et permet un deuxième accouplement pour les femelles non gravides à la suite du premier. Ainsi ménagés, les béliers de service ont une meilleure efficacité.

L'association d'un traitement progestagène et de l'effet mâle (Cognié 1988) est une perspective intéressante pour l'obtention d'une meilleure synchronisation des chaleurs et I'utilisation de l'insémination artificielle, sans avoir l'inconvénient d'utiliser une hormone protéique (eCG = equine Chorionic Gonadotropin) provenant d'une autre espèce (avec les risques sanitaires éventuels) et susceptible de former des anticorps diminuant la fertilité avec l'augmentation des traitements au cours de la carrière d'une femelle (Roy et al 1999). «L'injection» des béliers dans le troupeau de femelles immédiatement après l'arrêt du traitement progestagène est équivalente à une administration de eCG, tout en réduisant la proportion de portées supérieures ou égales à trois. Des inséminations artificielles sont possibles si des béliers vasectomisés sont utilisés. Le moment d'insémination par rapport au retrait du traitement progestagène doit cependant être modifié par rapport à un traitement classique, la présence des béliers suscitant une apparition plus précoce de l'ovulation (Cognié 1988, Lindsay et al 1975).

\section{2 / Règles d'utilisation}

- Les brebis doivent être séparées des mâles au moins un mois avant le début de la lutte. Elles ne doivent pas être mises dans une bergerie où les béliers ont séjourné, car leur «odeur» imprègne le bâtiment et la litière, et les parcs ou bergeries des mâles et des femelles doivent être aussi éloignés que possible.

- La date doit être choisie judicieusement. L'anoestrus est le moins intense lorsqu'il touche à son terme ce qui permet d'avancer la date de lutte quelle que soit la race. Pour les races du pourtour méditerranéen, la période d'efficacité est beaucoup plus large et peut être favorable dès la mi-avril. En revanche, certaines races des latitudes plus élevées, sont très peu sensibles à l'effet mâle car leur anoestrus est trop intense.

Les règles d'utilisation sont difficiles à donner pour chaque race. Toutefois, une très bonne indication, pour chaque race utilisée en France, est donnée par la répartition des agnelages dans les troupeaux soumis au Contrôle National des Performances (Perret 1986). Pour les races se reproduisant uniquement à l'automne (agnelages de printemps), l'effet mâle peut être efficace, chez les brebis adultes, 6,5 à 5 mois avant la date des premières mise bas enregistrées. Ce sont les races originaires du nord de la France ou les races d'origine britannique. Pour les autres races, des agnelages sont observés en automne: leur fréquence par rapport aux agnelages de printemps est variable d'une race à l'autre. Ces agnelages d'automne peuvent être liés à l'utilisation des traitements hormonaux d'induction de l'oestrus et de l'ovulation. Ils sont aussi les témoins d'une lutte à contre-saison par «effet mâle», de nombreux éleveurs pratiquant cette conduite de troupeau sans le savoir. Aux périodes de lutte correspondant à ces agnelages d'automne, un «effet mâle» sera efficace. Ces races sont plutôt originaires des régions sud de la France. Parmi elles, figurent aussi les différentes races Mérinos et celles ayant du sang Mérinos (exemple : race lle de France).

L'observation de la répartition des agnelages dans les différentes races montre que les mise bas des agnelles et/ou des antenaises sont plus tardives que celles des femelles adultes. Ceci reflète le plus fort saisonnement des jeunes femelles (anoestrus plus intense).

Sur un plan pratique, pour un éleveur n'ayant jamais utilisé l'effet mâle, il est conseillé de ne tenter l'expérience, dans un premier temps, que sur une partie des femelles adultes.

- Les femelles doivent être préparées à la lutte. Une suralimentation passagère (flushing), comme pour une lutte normale est souhaitable. Un bon état corporel correspondant à une note comprise entre 3 et 3,5 (Bocquier et al 1988) diminue l'intensité de l'anoestrus et améliore donc l'efficacité de l'effet mâle. II peut, aussi, accroître la prolificité. Pour les béliers, outre cette préparation alimentaire, qui doit être plus longue (environ deux mois) compte tenu de la durée de la spermatogenèse et de la durée du transit épididymaire des spermatozoïdes, une préparation plus spécifique peut être envisagée en ayant recours à des traitements photopério- 
diques associant ou non un traitement à la mélatonine. Des mâles ayant des capacités de reproduction identiques à celles qu'ils ont en saison normale de reproduction sont plus efficaces pour induire l'ovulation chez les femelles.

- Une tonte préalable des femelles (au moins trois semaines avant le début de la lutte) peut aussi être judicieuse. Traditionnellement, cette tonte était et est encore pratiquée par les éleveurs sans que son importance et son rôle soient clairement établis.

- Le nombre de béliers utilisés pour la lutte doit être modulé en fonction de l'afflux de brebis en chaleur et il convient de ne pas utiliser des béliers «déjà fatigués par des jouissances réitérées» (Girard 1813). L'élimination de certains béliers par un contrôle préalable de l'ardeur sexuelle et de la qualité des éjaculats améliore les résultats de lutte. Une meilleure gestion des mâles de reproduction est obtenue lorsque l'effet mâle est réalisé avec des béliers vasectomisés qui peuvent être laissés dans le troupeau pendant deux semaines avant d'être remplacés par les mâles reproducteurs.

- Pour une bonne gestion des béliers (vasectomisés et/ou reproducteurs) et ne pas augmenter leur nombre, lors d'une lutte libre sans contrôle des paternités, le troupeau de femelles peut être scindé en deux groupes qui seront rassemblés avec les mêmes mâles à un intervalle de 4 jours. Cet intervalle est le plus judicieux pour la répartition la plus uniforme possible des venues en oestrus pendant la période de lutte. La même procédure peut être effectuée lorsque l'éleveur desire connaître les paternités et, de ce fait, est obligé de conduire la lutte avec un seul bélier par lot. Un bélier pour 30 femelles semble être une bonne proportion. II reste que l'éleveur doit s'assurer de la qualité des béliers. L'utilisation de harnais marqueurs ou de tout autre moyen de détection des oestrus chez les femelles est là fortement conseillée.

Pendant trois années consécutives, sur un troupeau de race Mérinos d'Arles dont les effectifs ont varié de 327 à 357 femelles (adultes + antenaises), l'effet mâle a été utilisé pour induire une période d'activité sexuelle. Des béliers vasectomisés de race Mérinos d'Arles (une dizaine) ont servi comme inducteurs de l'ovulation chez les femelles anovulatoires. La moitié des femelles ont été mises en présence permanente de ces béliers vers le 12 avril. Les autres ont été introduites dans ce troupeau de béliers et de brebis 4 jours plus tard. Quatorze jours après la mise en contact du premier groupe de femelles avec les béliers vasectomisés, ceux-ci ont été remplacés par une vingtaine de béliers de race lle de France munis de harnais marqueurs pour effectuer la monte en bergerie, la nuit uniquement, au retour du pâturage des brebis (lutte en lot, sans connaissance des paternités). Les marques sur les femelles ont été enregistrées deux fois par semaine. La durée de la lutte proprement dite avec les béliers lle de France a varié de 37 à 40 jours suivant les années. Près de $90 \%$ des femelles ont été marquées par les béliers Ile de France munis de harnais marqueurs sur une période de 10 jours. Plus de $95 \%$ des femelles ont été marquées au moins une fois pendant la période de lutte. La fertilité lors du premier oestrus a varié selon les années de 78,4 à $82,9 \%(80,7 \%$ en moyenne sur les trois ans). Sur la période totale de lutte, elle a aussi légèrement varié d'une année à l'autre de 88,0 à $89,9 \%(89,1 \%$ en moyenne sur les trois ans). Ces résultats démontrent l'efficacité de l'effet mâle lorsqu'il est bien conduit. II a ainsi été possible de supprimer la lutte de rattrapage, habituelle en automne, dans les troupeaux Mérinos d'Arles.

\section{Conclusion}

L'effet mâle chez les ovins est une méthode pertinente et efficace pour induire, dans certaines limites toutefois, une periode de reproduction à contre-saison ou avancer la période de reproduction. Elle demande des moyens peu importants et son coût est extrêmement faible en lutte naturelle. Elle exige surtout la mobilisation des connaissances que les éleveurs et les techniciens ont sur les races qu'ils utilisent dans un milieu donné. Elle demande aussi le savoir-faire de l'éleveur. Beaucoup d'éleveurs pratiquent consciemment ou non l'effet mâle dans leur système de conduite du troupeau.

II est intéressant de souligner l'importance de l'analyse du «carnet d'agnelage» pour savoir si la répartition des mise bas après une lutte naturelle est:

- uniforme, ce qui indique que les femelles étaient normalement cycliques lors de la lutte ;

- non uniforme (figure 3) et reflète l'existence d'un effet mâle lors de la lutte. L'analyse de cette répartition est alors intéressante et permet de connaître beaucoup sur le passé du troupeau et de préciser l'intensité de l'anoestrus lors de la lutte. Si le premier pic de mise bas est plus important que le deuxième, l'anoestrus était peu intense. C'est l'inverse, anoestrus intense, si le deuxième pic est plus important que le premier.

Figure 3. Distribution temporelle des mise bas après effet mâle au printemps dans deux troupeaux de race Sicilo-sarde en Tunisie.

Le jour 1 correspond aux premières mise bas.

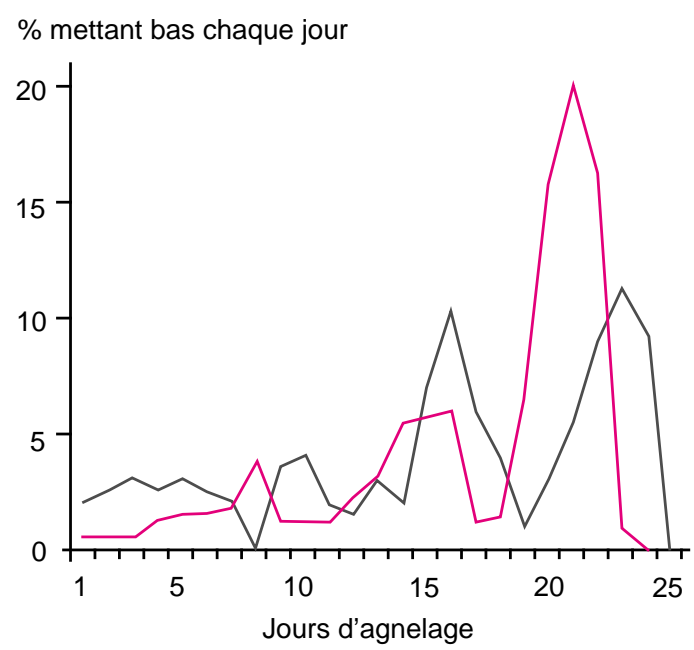

INRA Productions Ani males, octobre 2000 
Parmi les perspectives, trois aspects peuvent être indiqués :

- vérifier que, comme chez les caprins, la préparation des béliers en période de faible activité sexuelle par des traitements photopériodiques (lumière + mélatonine ou lumière seule) accroît la réponse des femelles à l'effet mâle ; - modéliser la réponse à l'effet mâle en fonction de différents paramètres (race des femelles et des mâles, activité sexuelle des mâles, intensité de l'anoestrus, période de l'année, état corporel, ....) permettant de prédire la réponse espérée :

- améliorer la réponse à l'effet mâle en modifiant l'intensité de l'anoestrus saisonnier par voie génétique. La récente mise en évidence du caractère reproductible et héritable de l'aptitude à ovuler spontanément au printemps (tout au moins en race Mérinos d'Arles : Hanocq et al 1998) et la liaison de cette aptitude avec un polymorphisme du récepteur de la mélatonine (Pelletier et al 2000), hormone très impliquée dans le saisonnement de la reproduction chez les petits ruminants, offrent de nouvelles possibilités.... à long terme en fonction de différents paramètres (race des femelles et des mâles, période de l'année, état corporel, ...).

II reste que la maîtrise des relations entre mâles et femelles et la maîtrise des facteurs alimentaires et nutritionnels au sein de l'élevage est le moyen plus efficace pour réussir une lutte par effet mâle.

\section{Références}

Bocquier F., Thériez M., Prache S., Brelurut A., 1988. Alimentation des ovins In : $R$. J arrige (ed) Alimentation des bovins, ovins et caprins, 249-279. INRA, Paris

Chemineau P., 1989. L'effet bouc : mode d'action et efficacité pour stimuler la reproduction des chèvres en anoestrus. INRA Prod. Anim., 2, 97-104

Chemineau P., Malpaux B., Delgadillo J.A., Guérin Y. Ravault J .P., Thimonier J., Pelletier J., 1992. Control of sheep and goat reproduction: use of light and melatonin. Anim. Reprod. Sci., 30, 157-184.

Chemineau P., Daveau A., Locatelli A., Maurice F., 1993. Ram-induced short luteal phases: effects of hysterectomy and cellular composition of the corpus luteum. Reprod. Nutr. Dev., 33, 253-261.

Chemineau P., Malpaux B., Pelletier J., Leboeuf B., Delgadillo J.A., Delétang F., Pobel T., Brice G. 1996 E mploi des implants de mélatonine et des traitements photopériodiques pour maîtriser la reproduction saisonnière chez les ovins et les caprins. INRA Prod. Anim., 9, 45-60.

Chemineau P., Baril G., Leboeuf B., Maurel M.C., Roy F., Pellicer-Rubio M., Malpaux B., Cognié Y., 1999. Implications des progrès récents en physiologie de la reproduction pour la conduite de la reproduction dans I'espèce caprine. INRA Prod. Anim., 12, 135-146.

Cognié Y., 1988. Nouvelles méthodes utilisées pour améliorer les performances de reproduction chez les ovins. INRA Prod. Anim., 1, 83-92.

Delgadillo J.A., Flores J.A., Velmiz F.G., Poindron P., Perez-Villanueva J .A., Martinez De La Escallera G., 2000. Photoperiodic treatment of bucks markedly improves the response of seasonally anovulatory goats to the «male effect».7ème Conférence Internationale sur les Caprins, 15-18 mai, Tours. INRA, International Goat Association et Institut de I'Elevage Eds, I, 396-399.

Girard L., 1813. Moyens employés avec succès, par M. Morel de Vindé, Membre de la Société d'Agriculture de Seine et Oise, pour obtenir, dans le temps le plus court possible, la fécondation du plus grand nombre des brebis portières d'un troupeau. Ephémérides de la Société d'Agriculture du Département de I'Indre pour I'An 1813, Séance du 5 Septembre, VIII Cahier, Château-Roux, Département de I'Indre, VII, 66-68.

Hanocq E., Bodin L., Thimonier J., Teyssier J ., Malpaux B., Chemineau P., 1998. Genetic parameters of spontaneous ovulatory activity in Merinos d'Arles sheep. Genet. Sel. Evol., 31, 77-90.
Khaldi G., Lassoued N., 1991. Interactions nutritionreproduction chez les petits ruminants en milieu méditerranéen. Proc. International Symposium on Nuclear and Related Techniques in Animal Production and Health. AIEA/FAO, 15-19 April, Vienna, 379-390.

Knight T.W., Lynch P.R., 1980. Source of ram pheromones that stimulate ovulation in the ewe. Anim. Reprod. Sci., 3, 133-136.

Knight T.W., Dalton D.C., Hight G.K., 1980. Changes in the median lambing dates and lambing pattern with variation in time of joining and breed of teasers. N-Z. J. Agric. Res., $23,281-285$

Lassoued N., Khaldi G., Cognié Y., Chemineau P. Thimonier J., 1995. Effet de la progestérone sur le taux d'ovulation et la durée du cycle ovarien induits par effet mâle chez la brebis Barbarine et la chèvre locale tunisienne. Reprod. Nutr. Dev., 35, 415-426.

Lassoued N., Khaldi G., Chemineau P., Cognié Y. Thimonier J., 1997. Role of the uterus in early regression of corpora lutea induced by the ram effect in seasonally anoestrous Barbarine ewes. Reprod. Nutr. Dev., 37, 559-571.

Lindsay D.R., Cognié Y., Pelletier I ., Signoret J.P., 1975 Influence of the presence of rams on the timing of ovula tion and discharge of LH in ewes. Physiol. Behav., 15, 423426.

Lindsay D.R., Cognié Y., Signoret J .P., 1982. Méthode simplifiée de maîtrise de l'oestrus chez la brebis. Ann. Zootech., 31, 77-82.

Oldham C.M., Lindsay D.R., 1980. Laparoscopy in the ewe: a photographic record of the ovarian activity of ewes experiencing normal or abnormal oestrous cycles. Anim. Reprod. Sci., 3, 119-124.

Ortavant R., Bocquier F., Pelletier J., Ravault J.P. Thimonier J., Volland-Nail P., 1988. Seasonality of reproduction in sheep and its control by photoperiod. Aust. J. Biol. Sci., 41, 69-85.

Pearce G.P., Oldham C.M., 1988. Importance of non-olfactory ram stimuli in mediating ram-induced ovulation in the ewe. J. Reprod. Fertil., 84, 333-339.

Pelletier J., Bodin L., Hanocq E., Malpaux B., Teyssier J., Thimonier J., Chemineau P., 2000. Association between expression of reproductive seasonality and alleles of the gene for $\mathrm{Mel}_{1 \mathrm{a}}$ receptor in the ewe. Biol. Reprod., 62, 1096-1101. 
Perret G., 1986. Les races ovines. Itovic Ed., Paris, 441 p.

Poindron P., Cognié Y., Gayerie F., Orgeur P., Oldham C.M., Ravault J.P., 1980. Changes in gonadotrophin and prolactin levels in isolated (seasonally or lactationally) anovular ewes with ovulation caused by the introduction of rams. Physiol. Behav., 25, 227-236.

Prud'hon M., Denoy I., 1969. Effets de l'introduction de béliers vasectomisés dans un troupeau Mérinos d'Arles 15 jours avant la lutte de printemps sur l'apparition des oestrus, la fréquence des erreurs de détection des ruts et la fertilité des brebis. Ann. Zootech., 18, 95-106.

Prud'hon M., Denoy I., Dauzier L., Desvignes A., 1966 Etude des résultats de six années d'élevage des brebis Mérinos d'Arles du Domaine du Merle. I. Le contrôle des ruts et sa validité. Ann. Zootech., 15, 123-133.

Prud'hon M., Denoy I., Desvignes A., Goussopoulos I., 1968. Etude des résultats de six années d'élevage des brebis Mérinos d'Arles du Domaine du Merle. II. Relations entre l'âge, le poids, l'époque de lutte des brebis et les divers paramètres de la fécondité. Ann. Zootech., 17, 3145

Prunier A., 1989. Influence de la présentation au verrat sur l'âge à la puberté des truies. INRA Prod. Anim., 2, 6572.

Robinson T.J., 1959. The estrous cycle of the ewe and doe. In : H.H. Cole et Cupps (eds), Reproduction in domestic Animals, 291-333. Academic Press, New-York.

Rouger Y., 1974. Etude des interactions de l'environnement et des hormones sexuelles dans la régulation du comportement sexuel des Bovidae. Thèse Doct. ès Sciences Naturelles, Université Rennes, 197 p.

Roy F., Combes B., Vaiman D., Cribiu E.P., Pobel T. Delétang F., Combarnous Y., Guillou F., Maurel M.C., 1999. Humoral immune response to equine chorionic gonadotropin in ewes: association with major histocompatibility complex and interference with subsequent fertility. Biol. Reprod., 61, 209-218.

Scaramuzzi R. ., Downing J A., 1999. Effect of progesterone on the GnRH-induced secretion of oestradiol and androstenedione from the autotransplanted ovary of the anoestrous ewe. J. Reprod. Fertil., 116, 127-132.

Signoret J.P., 1990. The influence of the ram effect on the breeding activity of ewes and its underlying physiology. In : C.M. Oldham, G.B. Martin and I.W. Purvis (eds), Reproductive Physiology of Merino Sheep. Concepts and Consequences, 59-70. School of Agriculture, The University of Western Australia, Nedlands, Perth.

Signoret J.P., Lévy F., Nowak R., Orgeur P., Schaal B., 1997. Le rôle de l'odorat dans les relations interindividuelles des animaux d'élevage. INRA Prod. Anim., 10, 339-348.

Southee J .A., Hunter M.G., Law A.S., Haresign W., 1988. Effect of hysterectomy on the short life cycle corpus luteum produced after $\mathrm{GnRH}$ induced abnormal corpora lutea in anoestrous ewes. J. Reprod. Fertil., 84, 149-155.

Tervit H.R., Havik P., Smith J.F., 1977. Effect of breed of ram on the onset of the breeding season in Romney ewes. Proc. N.-Z. Soc. Anim. Prod., 37, 142-148.

Thimonier J ., 2000. Détermination de l'état physiologique des femelles par analyse des niveaux de progestérone. NRA Prod. Anim., 13, 177-183.

Thimonier J., Mauléon P., 1969. Variations saisonnières du comportement d'oestrus et des activités ovarienne et hypophysaire chez les ovins. Ann. Biol. anim. Bioch. Biophys., 9, 233-250

Walkden-Brown S.W., Martin G.B., Restall B.J ., 1999. Role of male-female interactions in regulating reproduction in sheep and goats. . Reprod. Fertil., Suppl. 54, 243-257.

\section{Abstract}

The ram effect: an up-to-date method for the control of oestrus and ovulation in sheep.

The introduction of rams in a group of anoestrous females induces a silent ovulation (without oestrus) within 2 to 4 days. This silent ovulation is followed either by an ovarian cycle of "normal» duration (around 17 days) and a new ovulation time which is associated with oestrous behaviour, either by an ovarian cycle of short but constant ( 6 days) duration followed by a new silent ovulation and, after an ovarian cycle of normal duration, by and oestrus and ovulation

As a result, in female induced to ovulate, the first oestrus are observed either 18 to 20 days or 24 to 26 days after introduction of the rams in the flock.

Following mating, non pregnant females may return to oestrus except if the intensity of anoestrus is too strong at the time of these oestrus which are expected 1 to 1.5 month after the induced ovulation.
The point is made on the underlying physiological mechanisms involved in the process of this induction of ovulatory and oestrous activities by the ram effect. Practical rules for management of flocks that want to use this up-to-date technique of control of ovulation and oestrus in sheep are also given. The two main points are firstly the knowledge of seasonal variations of reproductive activity of females in their environment which determine the period of efficiency of the ram effect and, secondly, the opportunity and facilities to separate rams and females for a minimal period of one month before their physical contact for the «ram effect».

THIMONIER J., COGNIE Y., LASSOUED N., KHALDI G., 2000. L'effet mâle chez les ovins : une technique actuelle de maîtrise de la reproduction. INRA Prod. Anim., 13, 223-231. 